\title{
BI-OBJECTIVE LOCATION MODEL OF TWO RECTANGULAR FACILITIES
}

\author{
Masashi Miyagawa \\ University of Yamanashi
}

(Received September 30, 2020; Revised February 22, 2021)

\begin{abstract}
This paper develops a bi-objective model for determining the location and shape of two finitesize facilities. The objectives are to minimize both the closest and barrier distances. The former represents the accessibility of customers, whereas the latter represents the interference to travelers. The total closest and barrier distances are derived for two rectangular facilities in a rectangular city where the distance is measured as the rectilinear distance. The analytical expressions for the total closest and barrier distances demonstrate how the location and shape of the facilities affect the distances. A numerical example shows that there exists a tradeoff between the closest and barrier distances. The tradeoff curve provides planners with alternatives for the location and shape of the facilities. The Pareto optimal location and shape of the facilities are then obtained.
\end{abstract}

Keywords: Facility planning, finite-size facility, closest distance, barrier distance, rectilinear distance, Pareto optimal

\section{Introduction}

Facilities such as parks, stadiums, and cemeteries are represented as regions rather than points and called finite-size facilities. An important characteristic of finite-size facilities is that they act as barriers to travel because traveling within facilities is usually prohibited. Not only the accessibility of customers but also the interference to travelers should be considered when locating finite-size facilities.

The optimal location (placement) of finite-size facilities has been addressed. For circular facilities, Drezner [8] studied the Weber problem where both the facility and demand have circular shapes. Kurita [12] formulated the minisum and minimax location problems for a circular facility. For rectangular facilities, Carrizosa et al. [6] found the optimal location and shape of a rectangular facility that minimize the average distance to the demand set. Sarkar et al. [18] and Kelachankuttu et al. [11] considered the placement of a rectangular facility in the presence of existing facilities. Date et al. [7] proposed an efficient procedure for the placement problem of a rectangular facility. For arbitrary shaped facilities, Carrizosa et al. [5] considered a generalized Weber problem where both the facility and demand are represented as regions. Shiode [21] obtained the optimal shape and location of a polygonal facility. Savaş et al. [20] developed the facility placement problem for finding the location and orientation of a finite-size facility in the presence of barriers. Brimberg and Wesolowsky $[1,2,3]$ formulated the minisum and minimax location problems for a finite-size facility. Sarkar et al. [19] considered the facility placement problem with the center objective.

A limitation of the studies reviewed above is that they only focused on the accessibility of customers. The interference to travelers was also considered by Miyagawa [15], who presented an analytical model for determining the number and size of circular facilities. 
Miyagawa [14] proposed a bi-objective model for locating a rectangular facility so as to minimize both the closest distance of customers and the barrier distance of travelers. The model was extended by Miyagawa [16] to determine the size of the facility.

In this paper, we develop a bi-objective model for determining the location and shape of two rectangular facilities. The major characteristic of the model is that it considers both the accessibility of customers and the interference to travelers. The objectives are then to minimize both the closest and barrier distances. The former represents the accessibility of customers, whereas the latter represents the interference to travelers. The model extends the location model of a single facility by Miyagawa [14] to that of two facilities. This extension allows us to examine how the distance between facilities affects the closest and barrier distances, and whether two small facilities are better than one large facility. The present model will thus give an insight into further location models of finite-size facilities.

The remainder of this paper is organized as follows. The next section develops a location model of two rectangular facilities. Sections 3 and 4 derive the total closest and barrier distances, respectively. Section 5 provides a numerical example and considers a bi-objective problem where both the closest and barrier distances are minimized. The final section presents concluding remarks.

\section{Model}

Consider a rectangular city with side lengths $a_{1}$ and $a_{2}$, as shown in Figure 1 . The city has two facilities of the same shape represented as rectangles with side lengths $b_{1}$ and $b_{2}$. Let $W=b_{1} / b_{2}$ be the aspect ratio and $\left(x_{b}, y_{b}\right),\left(x_{b}^{\prime}, y_{b}^{\prime}\right)$ be the southwest corners of the facilities. The facilities act as barriers to travel and travelers need to make a detour to avoid the facilities.

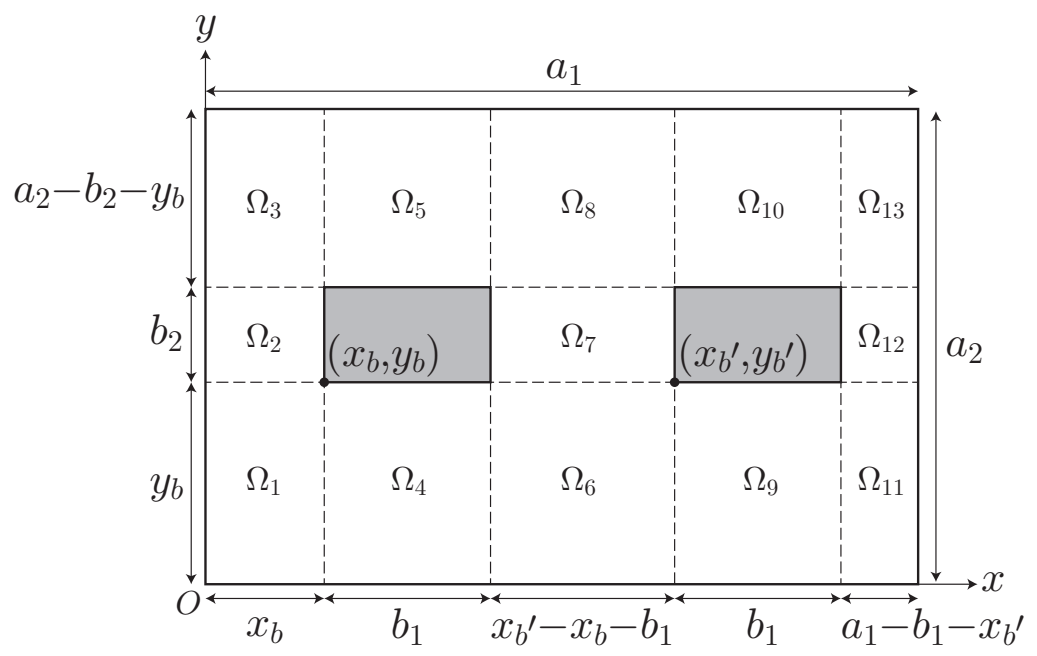

Figure 1: Two rectangular facilities in a rectangular city

The model involves the following assumptions. First, the two facilities have the same $y$-coordinate, i.e., $y_{b}=y_{b}^{\prime}$. Second, customers of the facilities are uniformly distributed in the city and use the nearest facility. Finally, origins and destinations of trips are also uniformly distributed. These assumptions yield analytical expressions for the closest and barrier distances, leading to a better understanding of fundamental relationships between variables. In addition, the result serves as a basis for further analysis with more generalized 
situations. For example, the travel demand depending on the trip length can be described by spatial interaction models [17].

The distance is measured as the rectilinear distance. The rectilinear distance between two points $\left(x_{1}, y_{1}\right)$ and $\left(x_{2}, y_{2}\right)$ is defined as $\left|x_{1}-x_{2}\right|+\left|y_{1}-y_{2}\right|$. The rectilinear distance is a good approximation for the actual travel distance in cities with a grid road network $[4,10,13]$.

\section{Closest Distance}

The closest distance is defined as the distance from customers to the closest point on the nearest facility. Customers in regions $\Omega_{1}-\Omega_{5}$ and the left half of regions $\Omega_{6}-\Omega_{8}$ use the left facility, whereas customers in the right half of regions $\Omega_{6}-\Omega_{8}$ and regions $\Omega_{9}-\Omega_{13}$ use the right facility in Figure 1. To obtain the total closest distance, we provide the rectilinear distance in a rectangle with side lengths $a$ and $b$, as shown in Figure 2. The sum of distances between a uniformly distributed point in the rectangle and a corner of the rectangle (Figure 2a) is

$$
\begin{aligned}
T_{1}(a, b) & =\int_{0}^{b} \int_{0}^{a}(x+y) \mathrm{d} x \mathrm{~d} y \\
& =\frac{a b}{2}(a+b) .
\end{aligned}
$$

The sum of distances between a uniformly distributed point in the rectangle and a side with length $b$ (Figure $2 \mathrm{~b}$ ) is

$$
\begin{aligned}
T_{2}(a, b) & =\int_{0}^{b} \int_{0}^{a} x \mathrm{~d} x \mathrm{~d} y \\
& =\frac{a^{2} b}{2} .
\end{aligned}
$$

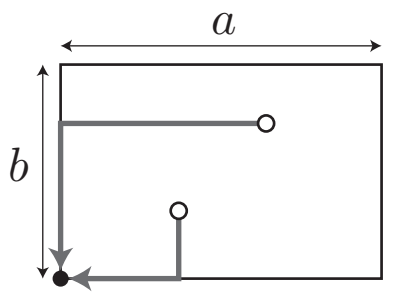

(a)

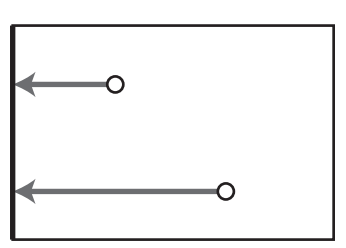

(b)

Figure 2: Calculation of the closest distance

Using Equations (3.1) and (3.2), we can obtain the total closest distance. For example, the total closest distances in regions $\Omega_{1}$ and $\Omega_{2}$ in Figure 1 are

$$
\begin{aligned}
& T_{1}\left(x_{b}, y_{b}\right)=\frac{x_{b} y_{b}}{2}\left(x_{b}+y_{b}\right), \\
& T_{2}\left(x_{b}, b_{2}\right)=\frac{x_{b}^{2} b_{2}}{2}
\end{aligned}
$$


respectively. The total closest distance in the city is then given by

$$
\begin{aligned}
T_{a}= & T_{1}\left(x_{b}, y_{b}\right)+T_{2}\left(x_{b}, b_{2}\right)+T_{1}\left(x_{b}, a_{2}-b_{2}-y_{b}\right)+2 T_{2}\left(y_{b}, b_{1}\right) \\
& +2 T_{2}\left(a_{2}-b_{2}-y_{b}, b_{1}\right)+2 T_{1}\left(\frac{x_{b}^{\prime}-x_{b}-b_{1}}{2}, y_{b}\right)+2 T_{2}\left(\frac{x_{b}^{\prime}-x_{b}-b_{1}}{2}, b_{2}\right) \\
& +2 T_{1}\left(\frac{x_{b}^{\prime}-x_{b}-b_{1}}{2}, a_{2}-b_{2}-y_{b}\right)+T_{1}\left(a_{1}-b_{1}-x_{b}^{\prime}, y_{b}\right) \\
& +T_{2}\left(a_{1}-b_{1}-x_{b}^{\prime}, b_{2}\right)+T_{1}\left(a_{1}-b_{1}-x_{b}^{\prime}, a_{2}-b_{2}-y_{b}\right) .
\end{aligned}
$$

\section{Barrier Distance}

The barrier distance is defined as the additional travel distance to avoid facilities, that is, the difference between the shortest distances in the presence and in the absence of facilities. The facilities hinders trips between regions $\Omega_{2}$ and $\Omega_{7}, \Omega_{2}$ and $\Omega_{12}, \Omega_{7}$ and $\Omega_{12}, \Omega_{4}$ and $\Omega_{5}$, and $\Omega_{9}$ and $\Omega_{10}$ in Figure 1. To obtain the total barrier distance, we provide the barrier distance between two uniformly distributed points in two rectangles, as shown in Figure 3a. Let $\left(x_{1}, y_{1}\right)$ and $\left(x_{2}, y_{2}\right)$ be two points uniformly distributed in the rectangles with side lengths $a_{1}$ and $b$ and with side lengths $a_{2}$ and $b$. The barrier distance is, if $y_{1} \leq y_{2}$,

$$
R= \begin{cases}2 y_{1}, & y_{1}+y_{2} \leq b, \\ 2\left(b-y_{2}\right), & y_{1}+y_{2}>b\end{cases}
$$

and if $y_{1}>y_{2}$,

$$
R= \begin{cases}2 y_{2}, & y_{1}+y_{2} \leq b, \\ 2\left(b-y_{1}\right), & y_{1}+y_{2}>b\end{cases}
$$

as shown in Figure 3b. Since Equations (4.1) and (4.2) are symmetric, the total barrier distance, denoted by $T\left(a_{1}, a_{2}, b\right)$, is obtained by considering only the case of $y_{1} \leq y_{2}$. Taking the round trip into account, we have

$$
\begin{aligned}
T\left(a_{1}, a_{2}, b\right)= & 4 \int_{0}^{b / 2} \int_{0}^{a_{1}} \int_{y_{1}}^{b-y_{1}} \int_{0}^{a_{2}} 2 y_{1} \mathrm{~d} x_{2} \mathrm{~d} y_{2} \mathrm{~d} x_{1} \mathrm{~d} y_{1} \\
& +4 \int_{0}^{b / 2} \int_{0}^{a_{1}} \int_{b-y_{1}}^{b} \int_{0}^{a_{2}} 2\left(b-y_{2}\right) \mathrm{d} x_{2} \mathrm{~d} y_{2} \mathrm{~d} x_{1} \mathrm{~d} y_{1} \\
& +4 \int_{b / 2}^{b} \int_{0}^{a_{1}} \int_{y_{1}}^{b} \int_{0}^{a_{2}} 2\left(b-y_{2}\right) \mathrm{d} x_{2} \mathrm{~d} y_{2} \mathrm{~d} x_{1} \mathrm{~d} y_{1} \\
= & \frac{2}{3} a_{1} a_{2} b^{3} .
\end{aligned}
$$

Using Equation (4.3), we can obtain the total barrier distance. For example, the total barrier distance of trips between regions $\Omega_{2}$ and $\Omega_{7}$ in Figure 1 is

$$
T\left(x_{b}, x_{b}^{\prime}-x_{b}-b_{1}, b_{2}\right)=\frac{2}{3} x_{b}\left(x_{b}^{\prime}-x_{b}-b_{1}\right) b_{2}^{3} .
$$

The total barrier distance in the city is then given by

$$
\begin{aligned}
T_{b}= & T\left(x_{b}, x_{b}^{\prime}-x_{b}-b_{1}, b_{2}\right)+T\left(x_{b}, a_{1}-b_{1}-x_{b}^{\prime}, b_{2}\right) \\
& +T\left(x_{b}^{\prime}-x_{b}-b_{1}, a_{1}-b_{1}-x_{b}^{\prime}, b_{2}\right)+2 T\left(y_{b}, a_{2}-b_{2}-y_{b}, b_{1}\right) .
\end{aligned}
$$




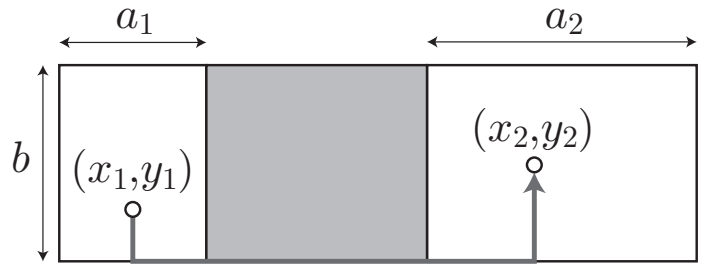

(a)

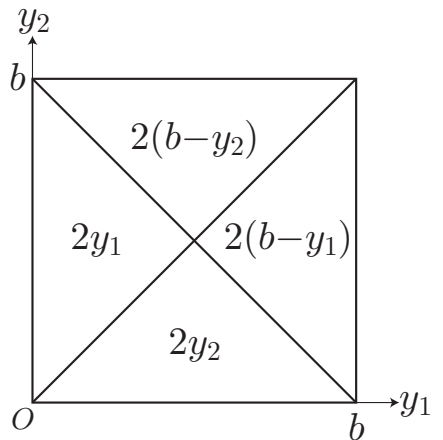

(b)

Figure 3: Calculation of the barrier distance

\section{Numerical Example}

As a numerical example, we consider three shapes of the facilities in square and rectangular cities, as shown in Table 1 . Note that the area of the facilities is always the same. The shapes of the square and rectangular cities are $a_{1}=a_{2}=1$ and $a_{1}=\sqrt{2}, a_{2}=1 / \sqrt{2}$, respectively. For the location of the facilities, we assume that $x_{b}^{\prime}=a_{1}-b_{1}-x_{b}$ and $y_{b}=\left(a_{2}-b_{2}\right) / 2$. The location of the two facilities for $x_{b}=0,\left(a_{1}-2 b_{1}\right) / 2$ are depicted in Figure 4 , where the two facilities become one large facility at $x_{b}=\left(a_{1}-2 b_{1}\right) / 2$.

Table 1: Shape of facility

\begin{tabular}{lll}
\hline Aspect ratio & $b_{1}$ & $b_{2}$ \\
\hline$W=2$ & 0.30 & 0.15 \\
$W=1$ & $0.15 \sqrt{2}$ & $0.15 \sqrt{2}$ \\
$W=1 / 2$ & 0.15 & 0.30 \\
\hline
\end{tabular}

The total closest distances for the three shapes of the facilities in the square and rectangular cities are shown in Figure 5. As the two facilities get closer with each other, the total closest distance first decreases and then increases. The optimal location of the facilities that minimizes the total closest distance is $x_{b}=\left(a_{1}-2 b_{1}\right) / 4$. This makes sense intuitively because the two facilities are at the centers of the left and right halves of the city. Among the three shapes of the facilities, $W=2$ is the best for small $x_{b}$ and $W=1 / 2$ is the best for large $x_{b}$. The total closest distance for $W=1$ is always greater than that for $W=2$ or $W=1 / 2$. It follows that the rectangular facilities are better than the square facilities in both the square and rectangular cities. In the square city, the minimum value for $W=1 / 2$ is smaller than that for $W=2$. In the rectangular city, the minimum value for $W=1 / 2$ is the same as that for $W=2$. Since the total closest distance depends on both the location and shape of the facilities, they should be determined simultaneously.

The total barrier distance for the three shapes of the facilities in the square and rectangular cities are shown in Figure 6. As the two facilities get closer with each other, the total barrier distance first increases and then decreases, and jumps at $x_{b}=\left(a_{1}-2 b_{1}\right) / 2$. Thus, two small facilities are better than one large facility. The optimal location of the facilities that minimizes the total barrier distance is $x_{b}=0$. This is because horizontal trips need not make a detour. In the square city, $W=1 / 2$ and $W=1$ are always better than $W=2$ except for $x_{b}=\left(a_{1}-2 b_{1}\right) / 2$. In the rectangular city, $W=1$ is almost always the best. In contrast to the closest distance case, the square facilities can be better than the rectangular 


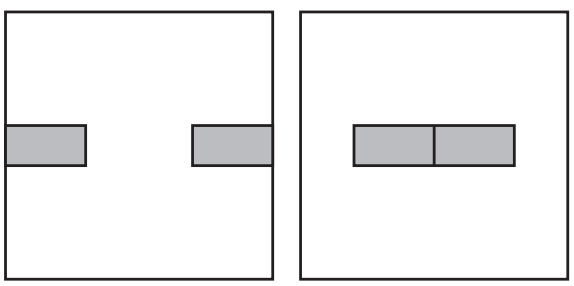

$W=2$

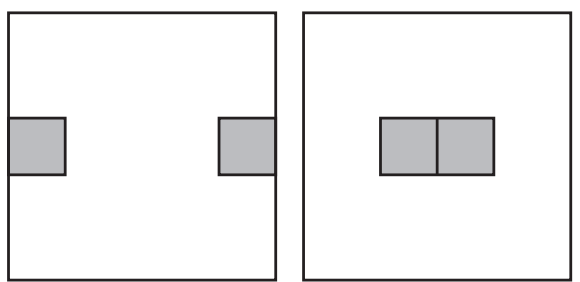

$W=1$

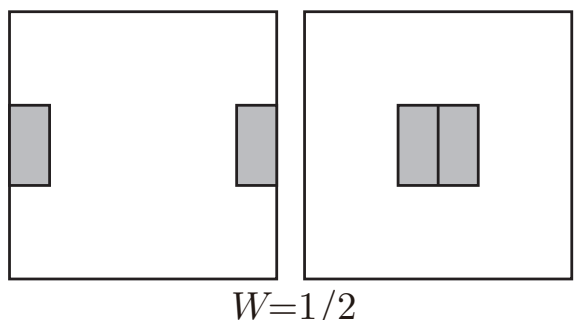

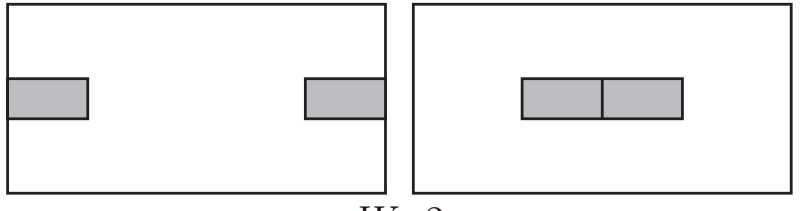

$W=2$
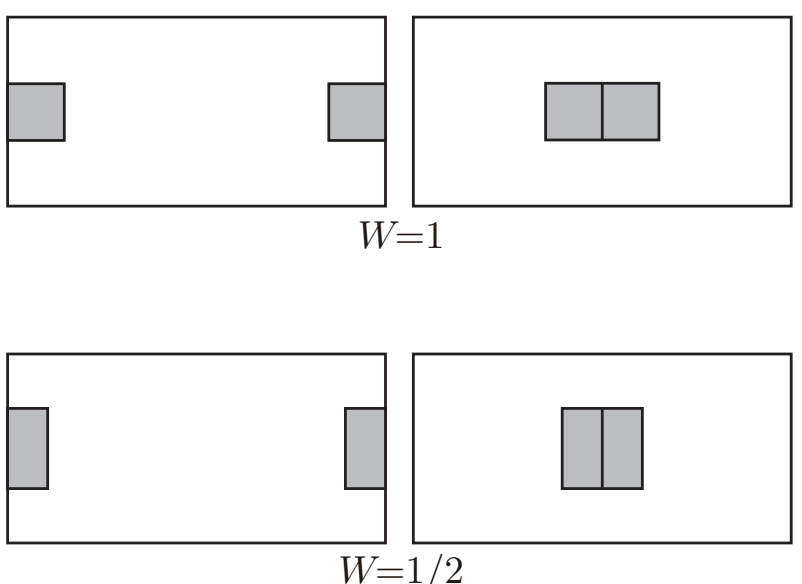

Figure 4: Three shapes of facilities in square and rectangular cities

facilities. The total barrier distance also depends on both the location and shape of the facilities.

Now, we consider a bi-objective problem where the total closest and barrier distances are minimized. We then obtain Pareto optimal solutions, which have frequently been used in multi-criteria facility location problems [9]. Pareto optimal solutions are such that no other solution is superior to them. For any two solutions $x$ and $y, x$ dominates $y$ if each criterion for $x$ is as good as that for $y$ and at least one criterion for $x$ is strictly better than that for $y$. The solution $x$ is called Pareto optimal if no feasible solution that dominates $x$ exists.

The total closest and barrier distances for $0<x_{b}<\left(a_{1}-2 b_{1}\right) / 2$ are shown in Figure 7 . If $0<x_{b}<\left(a_{1}-2 b_{1}\right) / 4$, as $x_{b}$ increases, the total closest distance decreases but the total barrier distance increases. Thus, there exists a tradeoff between the closest and barrier distances. It can be seen that the Pareto optimal location is $0<x_{b}<\left(a_{1}-2 b_{1}\right) / 4$. For the shape of the facilities, in the square city, $W=1 / 2$ is Pareto optimal because $W=1 / 2$ dominates both $W=2$ and $W=1$. In the rectangular city, $W=1$ is optimal in almost all the cases, but $W=2$ and $W=1 / 2$ can be optimal. In fact, $W=2$ and $W=1 / 2$ can minimize the total closest distance, and $W=1 / 2$ can minimize the total barrier distance.

\section{Conclusions}

This paper has presented a bi-objective location model of two finite-size facilities. The total closest and barrier distances have been derived for two rectangular facilities in a rectangular city. The location and shape of the facilities that minimize both the closest and barrier distances have then been obtained.

The model is useful for location analysis of finite-size facilities that considers both the 


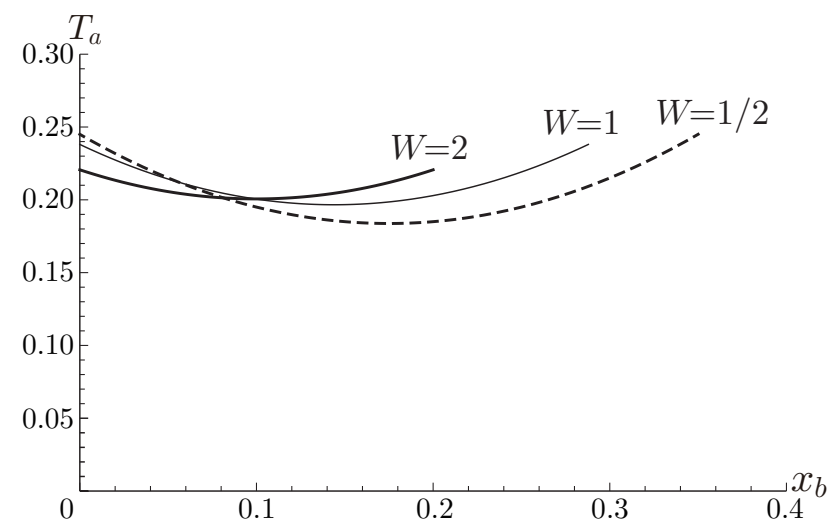

(a)

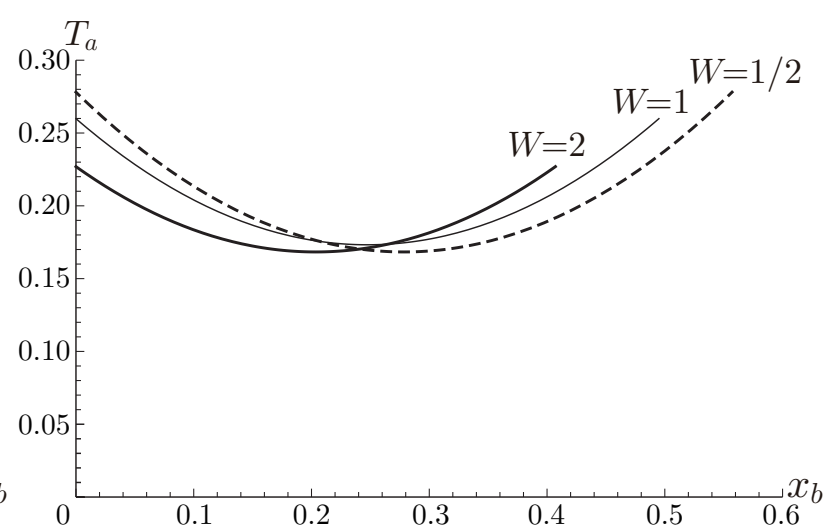

(b)

Figure 5: Total closest distance: (a) Square city; (b) Rectangular city

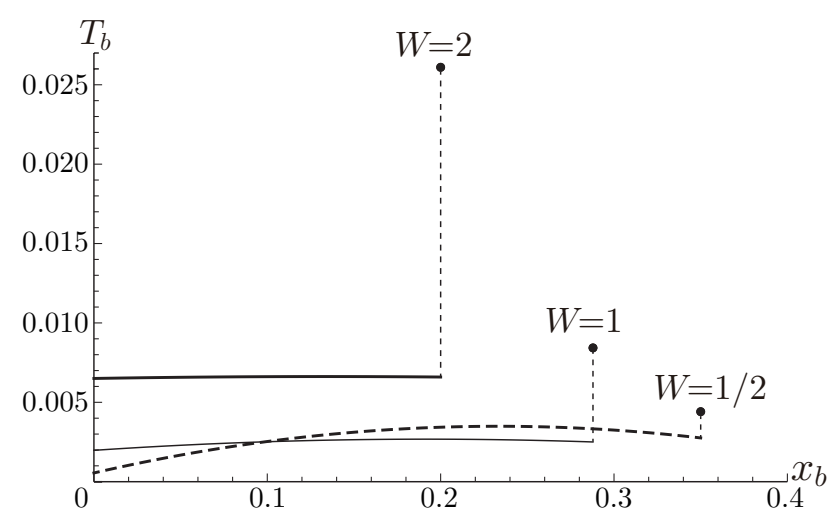

(a)

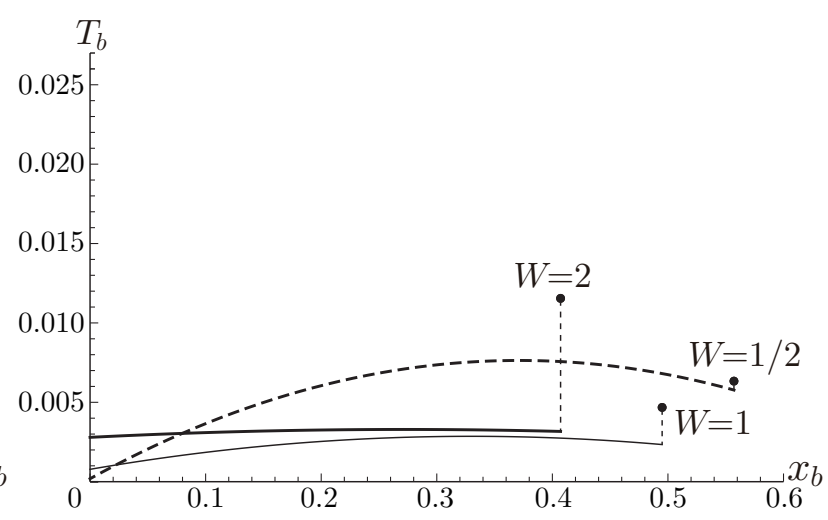

(b)

Figure 6: Total barrier distance: (a) Square city; (b) Rectangular city

accessibility of customers and the interference to travelers as follows. First, the analytical expressions for the total closest and barrier distances demonstrate how the location and shape of the facilities affect the distances. Note that finding these relationships by using empirical models requires computation of the distances for various combinations of the parameters. These relationships provide a fundamental understanding of the closest and barrier distances. Second, the model shows that there exists a tradeoff between the closest and barrier distances. The tradeoff curve provides planners with alternatives for the location and shape of the facilities. Finally, the Pareto optimal solutions can be used to evaluate actual location and shape of facilities and assist decisions about relocating and reshaping facilities.

An important topic for future research is the extension to more than two facilities. The result for two facilities in this paper suggests that if the total area of facilities is constant, both the closest and barrier distances decrease with the number of facilities. Thus, many small facilities would be better than few large facilities.

\section{Acknowledgements}

This research was supported by JSPS KAKENHI Grant Numbers JP18K04604, JP18K04628, JP19H02374. I am grateful to anonymous reviewers for their helpful comments and suggestions. 


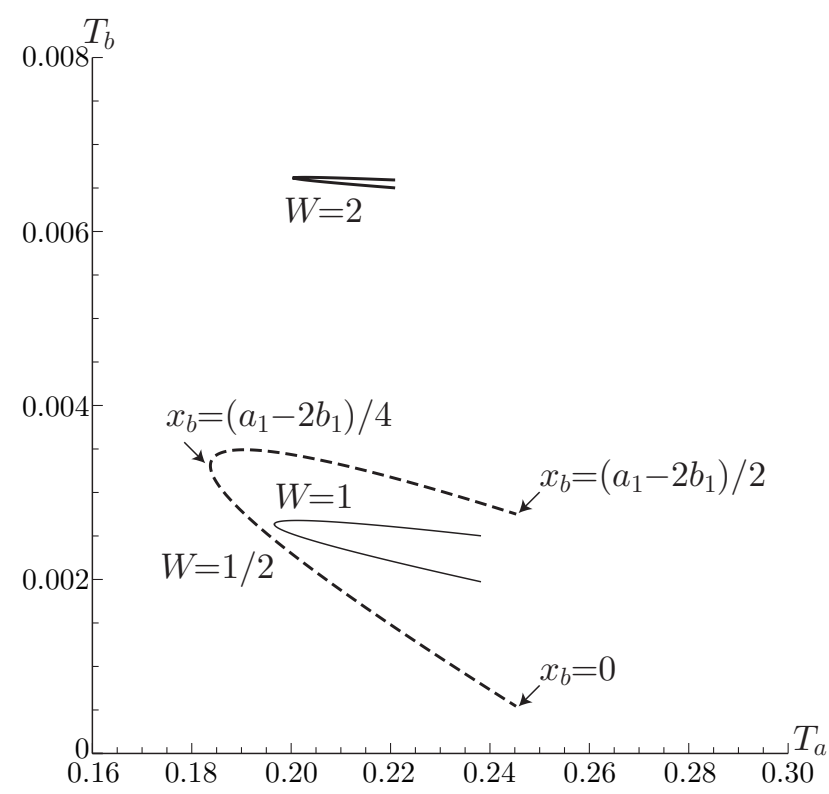

(a)

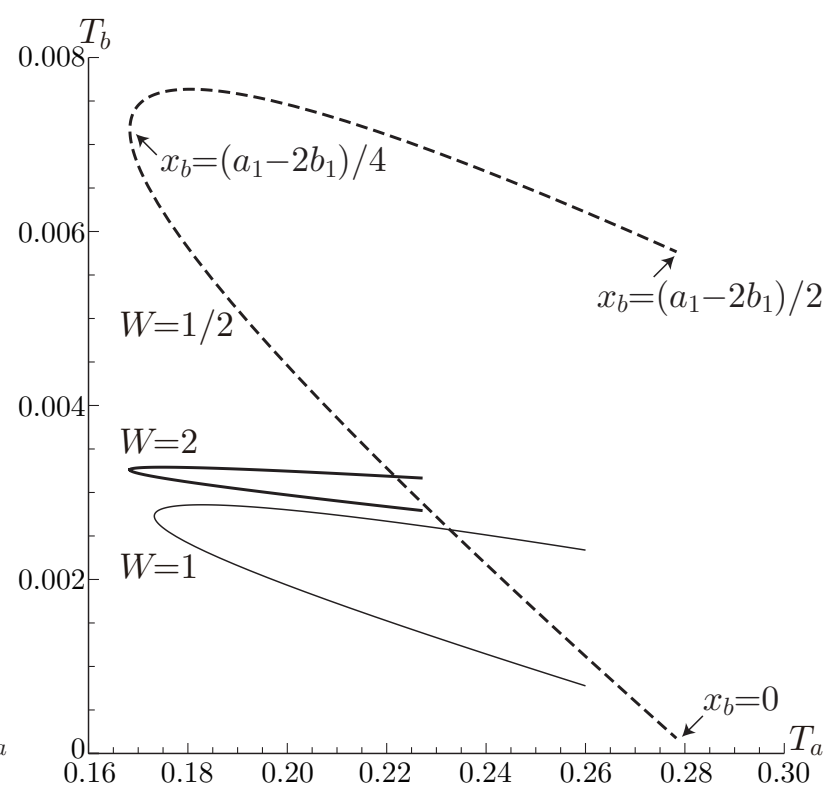

(b)

Figure 7: Total closest and barrier distances: (a) Square city; (b) Rectangular city

\section{References}

[1] J. Brimberg and G.O. Wesolowsky: Note: Facility location with closest rectangular distances. Naval Research Logistics, 47 (2000), 77-84.

[2] J. Brimberg and G.O. Wesolowsky: Locating facilities by minimax relative to closest points of demand areas. Computers \& Operations Research, 29 (2002), 625-636.

[3] J. Brimberg and G.O. Wesolowsky: Minisum location with closest Euclidean distances. Annals of Operations Research, 111 (2002), 151-165.

[4] J. Brimberg, J.H. Walker, and R.F. Love: Estimation of travel distances with the weighted $\ell_{p}$ norm: Some empirical results. Journal of Transport Geography, 15 (2007), $62-72$.

[5] E. Carrizosa, E. Conde, M. Muñoz-Márquez, and J. Puerto: The generalized Weber problem with expected distances. RAIRO Recherche Opérationnelle, 29 (1995), 35-57.

[6] E. Carrizosa, M. Muñoz-Márquez, and J. Puerto: Location and shape of a rectangular facility in $\mathbb{R}^{n}$. Convexity properties. Mathematical Programming, 83 (1998), 277-290.

[7] K. Date, S. Makked, and R. Nagi: Dominance rules for the optimal placement of a finite-size facility in an existing layout. Computers \& Operations Research, 51 (2014), $182-189$.

[8] Z. Drezner: Location of regional facilities. Naval Research Logistics Quarterly, 33 (1986), 523-529.

[9] R.Z. Farahani, M. SteadieSeifi, and N. Asgari: Multiple criteria facility location problems: A survey. Applied Mathematical Modelling, 34 (2010), 1689-1709.

[10] D.A. Griffith, I. Vojnovic, and J. Messina: Distances in residential space: Implications from estimated metric functions for minimum path distances. GIScience $\&$ Remote Sensing, 49 (2012), 1-30.

[11] H. Kelachankuttu, R. Batta, and R. Nagi: Contour line construction for a new rectangular facility in an existing layout with rectangular departments. European Journal of Operational Research, 180 (2007), 149-162. 
[12] O. Kurita: Location models for a facility with some area. Journal of the City Planning Institute of Japan, 26 (1991), 535-540 (in Japanese).

[13] R.F. Love and J.G. Morris: Mathematical models of road travel distances. Management Science, 25 (1979), 130-139.

[14] M. Miyagawa: Continuous location model of a rectangular barrier facility. TOP, 25 (2017), 95-110.

[15] M. Miyagawa: Bi-objective model for optimal number and size of circular facilities. Geographical Analysis, 49 (2017), 143-154.

[16] M. Miyagawa: Bi-objective model for optimal size and shape of a rectangular facility. Urban and Regional Planning Review, 4 (2017), 129-137.

[17] J.R. Roy: Spatial Interaction Modelling (Springer-Verlag, Berlin, 2010).

[18] A. Sarkar, R. Batta, and R. Nagi: Planar area location/layout problem in the presence of generalized congested regions with the rectilinear distance metric. IIE Transactions, 37 (2005), 35-50.

[19] A. Sarkar, R. Batta, and R. Nagi: Placing a finite size facility with a center objective on a rectangular plane with barriers. European Journal of Operational Research, 179 (2007), 1160-1176.

[20] S. Savaş, R. Batta, and R. Nagi: Finite-size facility placement in the presence of barriers to rectilinear travel. Operations Research, 50 (2002), 1018-1031.

[21] N. Shiode: A study on optimization of the shape and location of spatial facilities. Journal of the City Planning Institute of Japan, 30 (1995), 559-564 (in Japanese).

Masashi Miyagawa

Department of Regional Social Management University of Yamanashi

4-4-37 Takeda, Kofu

Yamanashi 400-8510, Japan

E-mail: mmiyagawa@yamanashi.ac.jp 